\title{
Editorial from the Editor in Chief
}

The topics addressed in this, the third issue of volume 25 are concerned with the generation of ultrashort, very intense pulses of laser radiation, and the relevant interaction processes with matter. Last year (2006) the international conference on the interaction of atoms, molecules, and pclasmas with intense ultrashort laser pulses covered these topics and we publish original papers that were submitted to Laser and Particle Beams after this conference. While most of these articles appear in this issue, two articles were already published (Stancalie et al., 2007; Ozaki et al., 2007).

With the September issue, we are also able to announce the new impact factor for Laser and Particle Beams. Each year in June, the journal citation reports section of the Thomson's ISI Web of Knowledge reports the new data about journal performance. Laser and Particle Beams impact factor improved to almost 4, and thus Laser and Particle Beams is the journal with the highest impact factor in plasma physics and applied plasma physics.

I want to take this opportunity to thank our authors for submitting their original papers to this journal and the editorial board for selecting the articles. The 10 top papers for Laser and Particle Beams listed in the essential scientific indicators are: Badziak et al., 2005; Glowacz et al., 2006; Hoffmann et al., 2005; Hora, 2004, 2005; Neumayer et al., 2005; Roth et al., 2005; Schaumann et al., 2005; the publications by Thareja \& Sharma, 2006 and Veiko et al., 2006 are also listed in the hot paper section.

\section{REFERENCES}

Badziak, J., Glowacz, S., Jablonski, S., Parys, P., Wolowski, J. \& Hora, H. (2005). Laser-driven generation of high-current ion beams using skin-layer ponderomotive acceleration. Laser Part. Beams 23, 401-409.

Glowacz, S., Hora, H., Badziak, J., Jablonski, S., Cang, Y. \& Osman, F. (2006). Analytical description of rippling effect and ion acceleration in plasma produced by a short laser pulse. Laser Part. Beams 24, 15-25.

Hoffmann, D.H.H., Blazevic, A., Ni, P., Rosmej, O., Roth, M., Tahir, N.A., Tauschwitz, A., Udrea, S., Varentsov, D.,
Weyrich, K. \& Maron, Y. (2005). Present and future perspectives for high energy density physics with intense heavy ion and laser beams. Laser Part. Beams 23, 47-53.

Hora, H. (2004). Developments in inertial fusion energy and beam fusion at magnetic confinement. Laser Part. Beams 22, 439-449.

Hora, H. (2005). Difference between relativistic petawattpicosecond laser-plasma interaction and subrelativistic plasmablock generation. Laser Part. Beams 23, 441-451.

Neumayer, P., Bock, R., Borneis, S., Brambrink, E., Brand, H., Caird, J., Campbell, E.M., Gaul, E., Goette, S., Haefner, C., Hahn, T., Heuck, H.M., Hoffmann, D.H.H., Javorkova, D., Kluge, H.J., Kuehl, T., Kunzer, S., Merz, T., Onkels, E., Perry, M.D., Reemts, D., Roth, M., Samek, S., Schaumann, G., Schrader, F., Seelig, W., Tauschwitz, A., Thiel, R., Ursescu, D., Wiewior, P., Wittrock, U. \& Zielbauer, B. (2005). Status of PHELIX laser and first experiments. Laser Part. Beams 23, 385-389.

Ozaki, T. Elouga Bom, L.B., Ganeev, R., KiefFer, J.-C., Suzuki, M. \& Kuroda, H. (2007). Intense harmonic generation from silver ablation. Laser Part. Beams 25, 321-325.

Stancalie, V., Pais, V., Tottolici, M. \& Mihailescu, A. (2007). Forbidden transitions in excitation by proton impact in Li-like Al ions. Laser Part. Beams 25, 277-282.

Roth, M., Brambrink, E., Audebert, P., Blazevic, A., Clarke, R., Cobble, J., Cowan, T.E., Fernandez, J., Fuchs, J., Geissel, M., Habs, D., Hegelich, M., Karsch, S., Ledingham, K., Neely, D., Ruhl, H., Schlegel, T. \& Schreiber, J. (2005). Laser accelerated ions and electron transport in ultra-intense laser matter interaction. Laser Part. Beams 23, 95-100.

Schaumann, G., Schollmeier, M.S., Rodriguez-Prieto, G., Blazevic, A., Brambrink, E., Geissel, M., Korostiy, S., Pirzadeh, P., Roth, M., Rosmej, F.B., Faenov, A.Y., Pikuz, T.A., Tsigutkin, K., Maron, Y., TAhir, NA., Hoffmann, D.H.H. (2005). High energy heavy ion jets emerging from laser plasma generated by long pulse laser beams from the NHELIX laser system at GSI. Laser Part. Beams 23 (4): 503-512.

Thareja, R.K. \& Sharma, A.K. (2006). Reactive pulsed laser ablation: Plasma studies. Laser Part. Beams 24, 311-320.

Veiko, V.P., Shakhno, E.A., Smirnov, V.N., Miaskovski, A.M. \& NiKISHIN, G.D. (2006). Laser-induced film deposition by LIFT: Physical mechanisms and applications. Laser Part. Beams 24, 203-209. 